\title{
Positive solutions for nonlocal fourth-order boundary value problems with all order derivatives
}

\author{
Yanping Guo ${ }^{1}$, Fei Yang ${ }^{2}$ and Yongchun Liang ${ }^{\text {* }}$
}

\footnotetext{
* Correspondence: lycocean@163. com

${ }^{1}$ College of Electrical Engineering and Information, Hebei University of Science and Technology, Shijiazhuang 050018, Hebei, P. R. China

Full list of author information is available at the end of the article
}

\begin{abstract}
In this article, by the fixed point theorem in a cone and the nonlocal fourth-order BVP's Green function, the existence of at least one positive solution for the nonlocal fourth-order boundary value problem with all order derivatives

$$
\left\{\begin{array}{l}
u^{(4)}(t)+A u^{\prime \prime}(t)=\lambda f\left(t, u(t), u^{\prime}(t), u^{\prime \prime}(t), u^{\prime \prime \prime}(t)\right), \quad 0<t<1 \\
u(0)=u(1)=\int_{0}^{1} p(s) u(s) d s \\
u^{\prime \prime}(0)=u^{\prime \prime}(1)=\int_{0}^{1} q(s) u^{\prime \prime}(s) d s
\end{array}\right.
$$

is considered, where $f$ is a nonnegative continuous function, $\lambda>0,0<A<\pi^{2}, p, q \in$ $L[0,1], p(s) \geq 0, q(s) \geq 0$. The emphasis here is that $f$ depends on all order derivatives.

Keywords: fourth-order boundary value problem, fixed point theorem, Green's function, positive solution
\end{abstract}

\section{Introduction}

The deformation of an elastic beam in equilibrium state, whose two ends are simply supported, can be described by a fourth-order ordinary equation boundary value problem. Owing to its significance in physics, the existence of positive solutions for the fourth-order boundary value problem has been studied by many authors using nonlinear alternatives of Leray-Schauder, the fixed point index theory, the Krasnosel'skii's fixed point theorem and the method of upper and lower solutions, in reference [1-10].

In recent years, there has been much attention on the question of positive solutions of the fourth-order differential equations with one or two parameters. By the Krasnosel'skii's fixed point theorem in cone [11], Bai [5] investigated the following fourthorder boundary value problem with one parameter

$$
\left\{\begin{array}{l}
u^{(4)}(t)+\beta u^{\prime \prime}(t)=\lambda f\left(t, u(t), u^{\prime \prime}(t)\right), \quad 0<t<1, \\
u(0)=u(1)=\int_{0}^{1} p(s) u(s) d s, \\
u^{\prime \prime}(0)=u^{\prime \prime}(1)=\int_{0}^{1} q(s) u^{\prime \prime}(s) d s,
\end{array}\right.
$$

where $\lambda>0,0<\beta<\pi^{2}, f: C([0,1] \times[0, \infty) \times(-\infty, 0],[0, \infty))$ is continuous, $p, q \in L[0,1]$, $p(s) \geq 0, q(s) \geq 0, \int_{0}^{1} p(s) d s<1, \int_{0}^{1} q(s) \sin \sqrt{\beta} s d s+\int_{0}^{1} q(s) \sin \sqrt{\beta}(1-s) d s<\sin \sqrt{\beta}$.

(C) 2012 Guo et al; licensee Springer. This is an Open Access article distributed under the terms of the Creative Commons Attribution License (http://creativecommons.org/licenses/by/2.0), which permits unrestricted use, distribution, and reproduction in any medium, provided the original work is properly cited. 
By the fixed point index in cone, Ma [7] proved the existence of symmetric positive solutions for the nonlocal fourth-order boundary value problem

$$
\left\{\begin{array}{l}
u^{(4)}(t)=h(t) f(t, u(t)), \quad 0<t<1, \\
u(0)=u(1)=\int_{0}^{1} p(s) u(s) d s, \\
u^{\prime \prime}(0)=u^{\prime \prime}(1)=\int_{0}^{1} q(s) u^{\prime \prime}(s) d s .
\end{array}\right.
$$

All the above works were done under the assumption that all order derivatives $u^{\prime}, u^{\prime \prime}$, $u^{\prime \prime \prime}$ are not involved explicitly in the nonlinear term $f$. In this article, we are concerned with the existence of positive solutions for the nonlocal fourth-order boundary value problem

$$
\left\{\begin{array}{l}
u^{(4)}(t)+A u^{\prime \prime}(t)=\lambda f\left(t, u(t), u^{\prime}(t), u^{\prime \prime}(t), u^{\prime \prime \prime}(t)\right), \quad 0<t<1, \\
u(0)=u(1)=\int_{0}^{1} p(s) u(s) d s \\
u^{\prime \prime}(0)=u^{\prime \prime}(1)=\int_{0}^{1} q(s) u^{\prime \prime}(s) d s
\end{array}\right.
$$

Throughout, we assume

$\left(H_{1}\right) \lambda>0,0<A<\pi^{2}$;

$\left(H_{2}\right) f:[0,1] \times R^{4} \rightarrow R^{+}$is continuous, $p, q \in L[0,1], p(s) \geq 0, q(s) \geq 0, \int_{0}^{1} p(s) d s<1$, $\int_{0}^{1} q(s) \sin \sqrt{A} s d s+\int_{0}^{1} q(s) \sin \sqrt{A}(1-s) d s<\sin \sqrt{A}$.

We will impose all order derivatives in $f$ and make use of two continuous convex functionals which will ensure the existence of at least one positive solution to (1.1). Bai [5] applied Krasnoselskii's fixed point theorem. Ma [8] used fixed point index in cone and Leray-Schauder degree. In this article, to show the existence of positive solutions to (1.1), we define two positive continuous convex functionals. Then, using the new fixed point theorem [12] in a cone and the nonlocal fourth-order BVP's Green function, we give some new criteria for the existence of positive solutions to (1.1).

\section{The preliminary lemmas}

Let $Y=C[0,1]$ be the Banach space equipped with the norm

$$
\|u(t)\|_{0}=\max _{t \in[0,1]}|u(t)| .
$$

Set $\lambda_{1}, \lambda_{2}$ be the roots of the polynomial $P(\lambda)=\lambda^{2}+A \lambda$, namely $\lambda_{1}=0, \lambda_{2}=-A$. By $\left(H_{1}\right)$, it is obviously that $-\pi^{2}<\lambda_{2}<0$.

Let $Q_{1}(t, s), Q_{2}(t, s)$ be, respectively the Green's functions of the following problems

$$
\left\{\begin{array} { l } 
{ - u ^ { \prime \prime } ( t ) + \lambda _ { 1 } u ( t ) = 0 , \quad 0 < t < 1 , } \\
{ u ( 0 ) = u ( 1 ) = \int _ { 0 } ^ { 1 } p ( s ) u ( s ) d s , }
\end{array} \quad \left\{\begin{array}{l}
-u^{\prime \prime}(t)+\lambda_{2} u(t)=0, \quad 0<t<1, \\
u(0)=u(1)=\int_{0}^{1} q(s) u(s) d s .
\end{array}\right.\right.
$$

Then, carefully calculation yield

$$
\begin{aligned}
& Q_{1}(t, s)=G_{1}(t, s)+\frac{\int_{0}^{1} G_{1}(s, x) p(x) d x}{1-\int_{0}^{1} p(x) d x}, \\
& Q_{2}(t, s)=G_{2}(t, s)+\frac{[\sin \sqrt{A} t+\sin \sqrt{A}(1-t)] \int_{0}^{1} G_{2}(s, x) q(x) d x}{\sin \sqrt{A}-\int_{0}^{1} q(x) \sin \sqrt{A} x d x-\int_{0}^{1} q(x) \sin \sqrt{A}(1-x) d x},
\end{aligned}
$$




$$
\begin{aligned}
& G_{1}(t, s)= \begin{cases}s(1-t), & 0 \leq s \leq t \leq 1, \\
t(1-s), & 0 \leq t \leq s \leq 1,\end{cases} \\
& G_{2}(t, s)= \begin{cases}\frac{\sin \sqrt{A} \sin \sqrt{A}(1-t)}{\sqrt{A} \sin \sqrt{A}}, & 0 \leq s \leq t \leq 1, \\
\frac{\sin \sqrt{A} t \sin \sqrt{A}(1-s)}{\sqrt{A} \sin \sqrt{A}}, & 0 \leq t \leq s \leq 1 .\end{cases}
\end{aligned}
$$

Denote

$$
\begin{aligned}
& \omega_{1}=\frac{1}{1-\int_{0}^{1} p(x) d x}, \\
& \omega_{2}(t)=\frac{\sin \sqrt{A} t+\sin \sqrt{A}(1-t)}{\sin \sqrt{A}-\int_{0}^{1} q(x) \sin \sqrt{A} x d x-\int_{0}^{1} q(x) \sin \sqrt{A}(1-x) d x} .
\end{aligned}
$$

Lemma 2.1. [5] Suppose that $\left(H_{1}\right)$ and $\left(H_{2}\right)$ hold. Then for any $y(t) \in C[0,1]$, the problem

$$
\left\{\begin{array}{l}
u^{(4)}(t)+A u^{\prime \prime}(t)=y(t), \quad 0<t<1, \\
u(0)=u(1)=\int_{0}^{1} p(s) u(s) d s \\
u^{\prime \prime}(0)=u^{\prime \prime}(1)=\int_{0}^{1} q(s) u^{\prime \prime}(s) d s .
\end{array}\right.
$$

has a unique solution

$$
u(t)=\int_{0}^{1} \int_{0}^{1} Q_{1}(t, s) Q_{2}(s, \tau) y(\tau) d \tau d s,
$$

where

$$
\begin{aligned}
& Q_{1}(t, s)=G_{1}(t, s)+\omega_{1} \int_{0}^{1} G_{1}(s, x) p(x) d x, \\
& Q_{2}(s, \tau)=G_{2}(s, \tau)+\omega_{2}(s) \int_{0}^{1} G_{2}(\tau, x) q(x) d x .
\end{aligned}
$$

By (2.2), we get

$$
\begin{aligned}
& u^{\prime}(t)=\int_{0}^{1} \int_{0}^{1} Q_{2}(s, \tau) y(\tau) d \tau d s-\int_{0}^{1} \int_{0}^{1} s Q_{2}(s, \tau) y(\tau) d \tau d s ; \\
& u^{\prime \prime}(t)=-\int_{0}^{1} Q_{2}(t, \tau) y(\tau) d \tau, \\
& u^{\prime \prime \prime}(t)=-\int_{0}^{1} \frac{\partial Q_{2}(t, \tau)}{\partial t} y(\tau) d \tau .
\end{aligned}
$$

Lemma 2.2. [5] Assume that $\left(H_{1}\right)$ and $\left(H_{2}\right)$ hold. Then one has

(i) $Q_{i}(t, s) \geq 0, \forall t, s \in[0,1] ; Q_{i}(t, s)>0, \forall t, s \in(0,1)$;

(ii) $G_{i}(t, s) \geq b_{i} G_{i}(t, t) G_{i}(s, s), \forall t, s \in[0,1]$;

(iii) $G_{i}(t, s) \leq c_{i} G_{i}(s, s), \forall t, s \in[0,1]$. 
where $b_{1}=1, b_{2}=\sqrt{A} \sin \sqrt{A} ; c_{1}=1, c_{2}=\frac{1}{\sin \sqrt{A}}$.

Let

$$
d_{i}=\min _{\frac{1}{4} \leq t \leq \frac{3}{4}} b_{i} G_{i}(t, t),(i=1,2) ; \xi=\frac{\min _{\frac{1}{4} \leq t \leq \frac{3}{4}} \omega_{2}(t)}{\max _{\frac{1}{4} \leq t \leq \frac{3}{4}} \omega_{2}(t)} .
$$

Lemma 2.3. [5] Suppose that $\left(H_{1}\right)$ and $\left(H_{2}\right)$ hold and $w_{2}, d_{i}, \xi_{i}$ are given as above.

\section{Then}

one has

(i) $\max _{0 \leq t \leq 1} \omega_{2}(t)=\omega_{2}\left(\frac{1}{2}\right)$;

(ii) $0<d_{i}<1, \quad 0<\xi<1$.

Lemma 2.4. If $y(t) \in C[0,1]$ and $y(t) \geq 0$, then the unique solution $u(t)$ of problem

satisfies

$$
\min _{\frac{1}{4} \leq t \leq \frac{3}{4}} u(t) \geq d_{1}\|u\|_{0}, \min _{\frac{1}{4} \leq t \leq \frac{3}{4}}\left(-u^{\prime \prime}(t)\right) \geq \frac{d_{2} \xi}{c_{2}}\left\|u^{\prime \prime}\right\|_{0} .
$$

Proof. By (2.2) and (iii) of Lemma 2.2, we get

$$
\begin{aligned}
u(t) & =\int_{0}^{1} \int_{0}^{1} Q_{1}(t, s) Q_{2}(s, \tau) y(\tau) d \tau d s \\
& \leq \int_{0}^{1} \int_{0}^{1}\left[c_{1} G_{1}(s, s)+\omega_{1} \int_{0}^{1} G_{1}(s, x) p(x) d x\right] Q_{2}(s, \tau) y(\tau) d \tau d s \\
& =\int_{0}^{1} \int_{0}^{1}\left[G_{1}(s, s)+\omega_{1} \int_{0}^{1} G_{1}(s, x) p(x) d x\right] Q_{2}(s, \tau) \gamma(\tau) d \tau d s \\
& =\int_{0}^{1} \int_{0}^{1} Q_{1}(s, s) Q_{2}(s, \tau) d \tau d s .
\end{aligned}
$$

So,

$$
\|u\|_{0} \leq \int_{0}^{1} \int_{0}^{1} Q_{1}(s, s) Q_{2}(s, \tau) d \tau d s .
$$

Using (ii) of Lemma 2.2, we have

$$
\begin{aligned}
\min _{\frac{1}{4} \leq t \leq \frac{3}{4}} u(t) & =\min _{\frac{1}{4} \leq t \leq \frac{3}{4}} \int_{0}^{1} \int_{0}^{1} Q_{1}(t, s) Q_{2}(s, \tau) \gamma(\tau) d \tau d s \\
& \geq \min _{\frac{1}{4} \leq t \leq \frac{3}{4}} \int_{0}^{1} \int_{0}^{1}\left[b_{1} G_{1}(t, t) G_{1}(s, s)\right. \\
& \left.+\omega_{1} \int_{0}^{1} G_{1}(s, x) p(x) d x\right] Q_{2}(s, \tau) \gamma(\tau) d \tau d s \\
& =\int_{0}^{1} \int_{0}^{1}\left[d_{1} G_{1}(s, s)+\omega_{1} \int_{0}^{1} G_{1}(s, x) p(x) d x\right] Q_{2}(s, \tau) y(\tau) d \tau d s \\
& \geq d_{1} \int_{0}^{1} \int_{0}^{1}\left[G_{1}(s, s)+\omega_{1} \int_{0}^{1} G_{1}(s, x) p(x) d x\right] Q_{2}(s, \tau) \gamma(\tau) d \tau d s \\
& =d_{1} \int_{0}^{1} \int_{0}^{1} Q_{1}(s, s) Q_{2}(s, \tau) \gamma(\tau) d \tau d s \\
& \geq d_{1}\|u\|_{0} .
\end{aligned}
$$


By (2:4) and (iii) of Lemma 2.2, we get

$$
\begin{aligned}
\max _{\frac{1}{4} \leq t \leq \frac{3}{4}}\left(-u^{\prime \prime}(t)\right) & =\max _{\frac{1}{4} \leq t \leq \frac{3}{4}} \int_{0}^{1} Q_{2}(t, \tau) y(\tau) d \tau \\
& \leq \int_{0}^{1}\left[c_{2} G_{2}(\tau, \tau)+\max _{\frac{1}{4} \leq t \leq \frac{3}{4}} \omega_{2}(t) \int_{0}^{1} G_{2}(\tau, x) q(x) d x\right] \gamma(\tau) d \tau \\
& \leq c_{2} \max _{\frac{1}{4} \leq t \leq \frac{3}{4}} \omega_{2}(t) \int_{0}^{1}\left[G_{2}(\tau, \tau)+\int_{0}^{1} G_{2}(\tau, x) q(x) d x\right] \gamma(\tau) d \tau
\end{aligned}
$$

So,

$$
\left\|u^{\prime \prime}\right\|_{0} \leq c_{2} \max _{\frac{1}{4} \leq t \leq \frac{3}{4}} \omega_{2}(t) \int_{0}^{1}\left[G_{2}(\tau, \tau)+\int_{0}^{1} G_{2}(\tau, x) q(x) d x\right] \gamma(\tau) d \tau .
$$

Using (ii) of Lemma 2.2, we have

$$
\begin{aligned}
\min _{\frac{1}{4} \leq t \leq \frac{3}{4}}\left(-u^{\prime \prime}(t)\right) & =\min _{\frac{1}{4} \leq t \leq \frac{3}{4}} \int_{0}^{1} Q_{2}(t, \tau) \gamma(\tau) d \tau \\
& \geq \min _{\frac{1}{4} \leq t \leq \frac{3}{4}} \int_{0}^{1}\left[b_{2} G_{2}(t, t) G_{2}(\tau, \tau)+\omega_{2}(t) \int_{0}^{1} G_{2}(\tau, x) q(x) d x\right] \gamma(\tau) d \tau \\
& \geq \int_{0}^{1}\left[b_{2} G_{2}(t, t) G_{2}(\tau, \tau)+\min _{\frac{1}{4} \leq t \leq \frac{3}{4}} \omega_{2}(t) \int_{0}^{1} G_{2}(\tau, x) q(x) d x\right] \gamma(\tau) d \tau \\
& =\int_{0}^{1}\left[d_{2} G_{2}(\tau, \tau)+\min _{\frac{1}{4} \leq t \leq \frac{3}{4}} \omega_{2}(t) \int_{0}^{1} G_{2}(\tau, x) q(x) d x\right] \gamma(\tau) d \tau \\
& \geq d_{2} \min _{\frac{1}{4} \leq t \leq \frac{3}{4}} \omega_{2}(t) \int_{0}^{1}\left[G_{2}(\tau, \tau)+\int_{0}^{1} G_{2}(\tau, x) q(x) d x\right] \gamma(\tau) d \tau \\
& \geq \frac{d_{2}}{c_{2}} \frac{\frac{1}{4} \leq t \leq \frac{3}{4}}{\max _{\frac{1}{4} \leq t \leq \frac{3}{4}} \omega_{2}(t)}\left\|u^{\prime \prime}\right\|_{0} \\
& \geq \frac{d_{2} \xi}{c_{2}}\left\|u^{\prime \prime}\right\|_{0} .
\end{aligned}
$$

The proof is completed.

Let $X$ be a Banach space and $K \subset X$ a cone. Suppose $\alpha, \beta: \times \rightarrow R^{+}$are two continuous convex functionals satisfying $\alpha(\lambda u)=|\lambda| \alpha(u), \beta(\lambda u)=|\lambda| \beta(u)$, for $u \in X, \lambda \in R$, and $\|u\| \leq M \max \{\alpha(u), \beta(u)\}$, for $u \in X$ and $\alpha(u) \leq \alpha(v)$ for $u, v \in K, u \leq v$, where $M$ $>0$ is a constant.

Theorem 2.1. [12] Let $r_{2}>r_{1}>0, L>0$ be constants and

$$
\Omega_{i}=\left\{u \in X: \alpha(u)<r_{i}, \beta(u)<L\right\}, \quad i=1,2,
$$

two bounded open sets in $X$. Set

$$
D_{i}=\left\{u \in X: \alpha(u)=r_{i}\right\}, \quad i=1,2 .
$$

Assume $T: K \rightarrow K$ is a completely continuous operator satisfying 
$\left(A_{1}\right) \alpha(T u)<r_{1}, u \in D_{1} \cap K ; \alpha(T u)>r_{2}, u \in D_{2} \cap K ;$

$\left(A_{2}\right) \beta(T u)<L, u \in K$

$\left(A_{3}\right)$ there is a $p \in\left(\Omega_{2} \cap K\right) \backslash\{0\}$ such that $\alpha(p) \neq 0$ and $\alpha(u+\lambda p) \geq \alpha(u)$, for all $u$ $\in K$ and $\lambda \geq 0$.

Then $T$ has at least one fixed point in $\left(\Omega_{2} \backslash \bar{\Omega}_{1}\right) \cap K$.

\section{The main results}

Let $X=C^{4}[0,1]$ be the Banach space equipped with the norm $\|u\|=\max _{t \in[0,1]}|u(t)|+\max _{t \in[0,1]}\left|u^{\prime}(t)\right|+\max _{t \in[0,1]}\left|u^{\prime \prime}(t)\right|+\max _{t \in[0,1]}\left|u^{\prime \prime \prime}(t)\right|, \quad$ and $K=\left\{u \in X: u(t) \geq 0, u^{\prime \prime}(t) \leq 0, \min _{\frac{1}{4} \leq t \leq \frac{3}{4}} u(t) \geq d_{1}\|u\|_{0}, \min _{\frac{1}{4} \leq t \leq \frac{3}{4}}\left(-u^{\prime \prime}(t)\right) \geq \frac{d_{2} \xi}{c_{2}}\left\|u^{\prime \prime}\right\|_{0}\right\}$ is a cone in $X$.

Define two continuous convex functionals $\alpha(u)=\max _{t \in[0,1]}|u(t)|+\max _{t \in[0,1]}\left|u^{\prime \prime}(t)\right|$ and $\beta(u)=\max _{t \in[0,1]}\left|u^{\prime}(t)\right|+\max _{t \in[0,1]}\left|u^{\prime \prime \prime}(t)\right|$, for each $u \in X$, then $\|u\| \leq 2 \max \{\alpha(u), \beta(u)\}$ and $\alpha(\lambda u)=|\lambda| \alpha(u), \beta(\lambda u)=|\lambda| \beta(u)$, for $u \in X, \lambda \in R ; \alpha(u) \leq \alpha(v)$ for $u, v \in K, u \leq v$.

In the following, we denote

$$
\begin{aligned}
B= & \int_{0}^{1} \int_{0}^{1} Q_{1}(s, s) Q_{2}(s, \tau) d \tau d s, \\
D= & \int_{0}^{1}\left[G_{2}(\tau, \tau)+\omega_{2}\left(\frac{1}{2}\right) \int_{0}^{1} G_{2}(\tau, x) q(x) d x\right] d \tau, \\
F= & \frac{1}{\sin \sqrt{A}} \int_{0}^{1} \sin \sqrt{A} \tau d \tau \\
& +\frac{\sqrt{A} \int_{0}^{1} \int_{0}^{1} G_{2}(\tau, x) q(x) d x d \tau}{\sin \sqrt{A}-\int_{0}^{1} q(x) \sin \sqrt{A} x d x-\int_{0}^{1} q(x) \sin \sqrt{A}(1-x) d x}, \\
\eta_{0}= & \frac{1}{B+c_{2} D^{\prime}}, \eta_{1}=\frac{1}{\int_{\frac{1}{4}}^{\frac{3}{4}} Q_{2}\left(\frac{1}{2}, \tau\right) d \tau}, \eta_{2}=\frac{2}{3 c_{2} D+4 F}, \theta=\min \left\{\frac{d_{1}}{2}, \frac{d_{2} \xi}{2 c_{2}}\right\} .
\end{aligned}
$$

We will suppose that there are $L>b>\theta b>c>0$ such that $f\left(t, u, v, u_{0}, v_{0}\right)$ satisfies the following growth conditions:

$$
\begin{aligned}
&\left(H_{3}\right) f\left(t, u, v, u_{0}, v_{0}\right)<\frac{c \eta_{0}}{\lambda}, \text { for }\left(t, u, v, u_{0}, v_{0}\right) \in[0,1] \times[0, c] \times[-L, L] \times[-c, 0] \times[-L, L], \\
&\left(H_{4}\right) f\left(t, u, v, u_{0}, v_{0}\right) \geq \frac{b \eta_{1}}{\lambda}, \text { for }\left(t, u, v, u_{0}, v_{0}\right) \in\left[\frac{1}{4}, \frac{3}{4}\right] \times[\theta b, b] \times[-L, L] \times[-b, 0] \times[-L, L] \\
& \cup\left[\frac{1}{4}, \frac{3}{4}\right] \times[0, b] \times[-L, L] \times[-b,-\theta b] \times[-L, L], \\
&\left(H_{5}\right) f\left(t, u, v, u_{0}, v_{0}\right)<\frac{L \eta_{2}}{\lambda}, \text { for }\left(t, u, v, u_{0}, v_{0}\right) \in[0,1] \times[0, b] \times[-L, L] \times[-b, 0] \times[-L, L] .
\end{aligned}
$$

Let $f_{1}\left(t, u, v, u_{0}, v_{0}\right)=f_{1}\left(t, u^{*}, v^{*}, u_{0}^{*}, v_{0}^{*}\right)$, where

$$
\begin{aligned}
& u^{*}=\min \{\max (u, 0), b\}, v^{*}=\min \{\max (v,-\mathrm{L}), \mathrm{L}\}, \\
& u_{0}^{*}=\min \left\{\max \left(u_{0},-b\right), 0\right\}, v_{0}^{*}=\min \{\max (v,-L), L\} .
\end{aligned}
$$

We denote

$$
(T u)(t)=\lambda \int_{0}^{1} \int_{0}^{1} Q_{1}(t, s) Q_{2}(s, \tau) f_{1}\left(\tau, u(\tau), u^{\prime}(\tau), u^{\prime \prime}(\tau), u^{\prime \prime \prime}(\tau)\right) d \tau d s,
$$


$\begin{aligned}(T u)^{\prime}(t)= & \lambda\left[\int_{t}^{1} \int_{0}^{1} Q_{2}(s, \tau) f_{1}\left(\tau, u(\tau), u^{\prime}(\tau), u^{\prime \prime}(\tau), u^{\prime \prime \prime}(\tau)\right) d \tau d s\right. \\ & \left.-\int_{0}^{1} \int_{0}^{1} s Q_{2}(s, \tau) f_{1}\left(\tau, u(\tau), u^{\prime}(\tau), u^{\prime \prime}(\tau), u^{\prime \prime \prime}(\tau)\right) d \tau d s\right],\end{aligned}$

$(T u)^{\prime \prime}(t)=-\lambda \int_{0}^{1} Q_{2}(t, \tau) f_{1}\left(\tau, u(\tau), u^{\prime}(\tau), u^{\prime \prime}(\tau), u^{\prime \prime \prime}(\tau)\right) d \tau$,

$(T u)^{\prime \prime \prime}(t)=-\lambda \int_{0}^{1} \frac{\partial Q_{2}(t, \tau)}{\partial t} f_{1}\left(\tau, u(\tau), u^{\prime}(\tau), u^{\prime \prime}(\tau), u^{\prime \prime \prime}(\tau)\right) d \tau$

Lemma 3.1. Suppose that $\left(H_{1}\right)$ and $\left(H_{2}\right)$ hold. Then $T: K \rightarrow K$ is completely continuous.

Proof. For $u \in K$, by (3.1), (3.3) and Lemma 2.2, it is obviously that $T u \geq 0$, $(T u)^{\prime \prime} \leq$ 0 . In view of $c_{1}=1, c_{2}>1$, so

$$
\begin{aligned}
& \|\mathrm{Tu}\|_{0}=\max _{t \in[0,1]}\left|\lambda \int_{0}^{1} \int_{0}^{1} Q_{1}(t, s) Q_{2}(s, \tau) f_{1}\left(t, u(\tau), u^{\prime}(\tau), u^{\prime \prime}(\tau), u^{\prime \prime \prime}(\tau)\right) d \tau d s\right| \\
& \leq \lambda \int_{0}^{1} \int_{0}^{1}\left[c_{1} G_{1}(s, s)\right. \\
& \left.+\omega_{1} \int_{0}^{1} G_{1}(s, x) p(x) d x\right] Q_{2}(s, \tau) f_{1}\left(\tau, u(\tau), u^{\prime}(\tau), u^{\prime \prime}(\tau), u^{\prime \prime \prime}(\tau)\right) d \tau d s \\
& =\lambda \int_{0}^{1} \int_{0}^{1} Q_{1}(s, s) Q_{2}(s, \tau) f_{1}\left(\tau, u(\tau), u^{\prime}(\tau), u^{\prime \prime}(\tau), u^{\prime \prime \prime}(\tau)\right) d \tau d s, \\
& \left\|(T u)^{\prime \prime}\right\|_{0}=\max _{t \in[0,1]}\left|-\lambda \int_{0}^{1} Q_{2}(t, \tau) f_{1}\left(\tau, u(\tau), u^{\prime}(\tau), u^{\prime \prime}(\tau), u^{\prime \prime \prime}(\tau)\right) d \tau\right| \\
& \leq \lambda \int_{0}^{1}\left[c_{2} G_{2}(\tau, \tau)\right. \\
& \left.+\max _{\frac{1}{4} \leq t \leq \frac{3}{4}} \omega_{2}(t) \int_{0}^{1} G_{2}(\tau, x) q(x) d x\right] f_{1}\left(\tau, u(\tau), u^{\prime}(\tau), u^{\prime \prime}(\tau), u^{\prime \prime \prime}(\tau)\right) d \tau \\
& \leq \max _{\frac{1}{4} \leq t \leq \frac{3}{4}} \omega_{2}(t) \int_{0}^{1}\left[G_{2}(\tau, \tau)+\int_{0}^{1} G_{2}(\tau, x) q(x) d x\right] \\
& \times f_{1}\left(\tau, u(\tau), u^{\prime}(\tau), u^{\prime \prime}(\tau), u^{\prime \prime \prime}(\tau)\right) d \tau \text {. }
\end{aligned}
$$


By Lemma 2.3, (3.1) and (3.3), we have

$$
\begin{aligned}
& \min _{\frac{1}{4} \leq t \leq \frac{3}{4}}(T u)(t)=\min _{\frac{1}{4} \leq t \leq \frac{3}{4}} \lambda \int_{0}^{1} \int_{0}^{1} Q_{1}(t, s) Q_{2}(s, \tau) f_{1}\left(\tau, u(\tau), u^{\prime}(\tau), u^{\prime \prime}(\tau), u^{\prime \prime \prime}(\tau)\right) d \tau d s \\
& \geq \lambda \int_{0}^{1} \int_{0}^{1}\left[b_{1} G_{1}(t, t) G_{1}(s, s)\right. \\
& \left.+\omega_{1} \int_{0}^{1} G_{1}(s, x) p(x) d x\right] Q_{2}(s, \tau) f_{1}\left(\tau, u(\tau), u^{\prime}(\tau), u^{\prime \prime}(\tau), u^{\prime \prime \prime}(\tau)\right) d \tau d s \\
& =\lambda \int_{0}^{1} \int_{0}^{1}\left[d_{1} G_{1}(s, s)+\omega_{1} \int_{0}^{1} G_{1}(s, x) p(x) d x\right] \\
& \times Q_{2}(s, \tau) f_{1}\left(\tau, u(\tau), u^{\prime}(\tau), u^{\prime \prime}(\tau), u^{\prime \prime \prime}(\tau)\right) d \tau d s \\
& \geq d_{1} \lambda \int_{0}^{1} \int_{0}^{1} Q_{1}(s, s) Q_{2}(s, \tau) f_{1}\left(\tau, u(\tau), u^{\prime}(\tau), u^{\prime \prime}(\tau), u^{\prime \prime \prime}(\tau)\right) d \tau d s \\
& \geq d_{1}\|T u\|_{0}, \\
& \min _{\frac{1}{4} \leq t \leq \frac{3}{4}}\left(-(T u)^{\prime \prime}(t)\right)=\min _{\frac{1}{4} \leq t \leq \frac{3}{4}} \int_{0}^{1} Q_{2}(t, \tau) f_{1}\left(\tau, u(\tau), u^{\prime}(\tau), u^{\prime \prime}(\tau), u^{\prime \prime \prime}(\tau)\right) d \tau \\
& \geq \min _{\frac{1}{4} \leq t \leq \frac{3}{4}} \int_{0}^{1}\left[b_{2} G_{2}(t, t) G_{2}(\tau, \tau)\right. \\
& \left.+\omega_{2}(t) \int_{0}^{1} G_{2}(\tau, x) q(x) d x\right] f_{1}\left(\tau, u(\tau), u^{\prime}(\tau), u^{\prime \prime}(\tau), u^{\prime \prime \prime}(\tau)\right) d \tau \\
& \geq \int_{0}^{1}\left[b_{2} G_{2}(t, t) G_{2}(\tau, \tau)\right. \\
& \left.+\min _{\frac{1}{4} \leq t \leq \frac{3}{4}} \omega_{2}(t) \int_{0}^{1} G_{2}(\tau, x) q(x) d x\right] f_{1}\left(\tau, u(\tau), u^{\prime}(\tau), u^{\prime \prime}(\tau), u^{\prime \prime \prime}(\tau)\right) d \tau \\
& =\int_{0}^{1}\left[d_{2} G_{2}(\tau, \tau)\right. \\
& \left.+\min _{\frac{1}{4} \leq t \leq \frac{3}{4}} \omega_{2}(t) \int_{0}^{1} G_{2}(\tau, x) q(x) d x\right] f_{1}\left(\tau, u(\tau), u^{\prime}(\tau), u^{\prime \prime}(\tau), u^{\prime \prime \prime}(\tau)\right) d \tau \\
& \geq d_{2} \min _{\frac{1}{4} \leq t \leq \frac{3}{4}} \omega_{2}(t) \int_{0}^{1}\left[G_{2}(\tau, \tau)\right. \\
& \left.+\int_{0}^{1} G_{2}(\tau, x) q(x) d x\right] f_{1}\left(\tau, u(\tau), u^{\prime}(\tau), u^{\prime \prime}(\tau), u^{\prime \prime \prime}(\tau)\right) d \tau \\
& \geq \frac{d_{2}}{c_{2}} \frac{\min _{\frac{1}{4} \leq t \leq \frac{3}{4}} \omega_{2}(t)}{\max _{\frac{1}{4} \leq t \leq \frac{3}{4}} \omega_{2}(t)}\left\|(T u)^{\prime \prime}\right\|_{0} \\
& \geq \frac{d_{2} \xi}{c_{2}}\left\|(T u)^{\prime \prime}\right\|_{0} \text {. }
\end{aligned}
$$

So we can get $T(K) \subset K$ : Let $B \subset K$ is bounded, it is clear that $T(B)$ is bounded. Using $f_{1}, Q_{1}(t, s), Q_{2}(t, s)$ is continuous, we show that $T(B)$ is equicontinuous. By the Arzela-Ascoli theorem, a standard proof yields $T: K \rightarrow K$ is completely continuous.

Theorem 3.1. Suppose that $\left(H_{1}\right)-\left(H_{5}\right)$ hold. Then BVP $(1.1)$ has at least one positive solution $u(t)$ satisfying

$$
c<\alpha(u)<b, \quad \beta(u)<L .
$$

Proof. Take

$$
\Omega_{1}=\{u \in X: \alpha(u)<c, \quad \beta(u)<L\}, \quad \Omega_{2}=\{u \in X: \alpha(u)<b, \quad \beta(u)<L\},
$$


two bounded open sets in $X$, and

$$
D_{1}=\{u \in X: \alpha(u)=c\}, \quad D_{2}=\{u \in X: \alpha(u)=b\} .
$$

By Lemma 3.1, $T: K \rightarrow K$ is completely continuous. Let $p=\frac{b}{2} \in\left(\Omega_{2} \cap K\right) \backslash\{0\}, \alpha(p) \neq 0$. It is easy to see that $\alpha(u+\lambda p) \geq \alpha(u)$, for all $u \in K$ and $\lambda \geq 0$.

Let $u \in D_{1}$, we have

$$
\begin{aligned}
\|T u\|_{0}= & \max _{t \in[0,1]}\left|\lambda \int_{0}^{1} \int_{0}^{1} Q_{1}(t, s) Q_{2}(s, \tau) f_{1}\left(\tau, u(\tau), u^{\prime}(\tau), u^{\prime \prime}(\tau), u^{\prime \prime \prime}(\tau)\right) d \tau d s\right| \\
\leq & \lambda \int_{0}^{1} \int_{0}^{1}\left[c_{1} G_{1}(s, s)+\omega_{1} \int_{0}^{1} G_{1}(s, x) p(x) d x\right] Q_{2}(s, \tau) d \tau d s \times \frac{c \eta_{0}}{\lambda} \\
= & c \eta_{0} \int_{0}^{1} \int_{0}^{1} Q_{1}(s, s) Q_{2}(s, \tau) d \tau d s \\
= & B c \eta_{0}, \\
\left\|(T u)^{\prime \prime}\right\|_{0} & =\max _{t \in[0,1]}\left|-\lambda \int_{0}^{1} Q_{2}(t, \tau) f_{1}\left(\tau, u(\tau), u^{\prime}(\tau), u^{\prime \prime}(\tau), u^{\prime \prime \prime}(\tau)\right) d \tau\right| \\
& <\lambda \int_{0}^{1}\left[c_{2} G_{2}(\tau, \tau)+\omega_{2}\left(\frac{1}{2}\right) \int_{0}^{1} G_{2}(\tau, x) q(x) d x\right] d \tau \times \frac{c \eta_{0}}{\lambda} \\
& \leq c_{2} c \eta_{0} \int_{0}^{1}\left[G_{2}(\tau, \tau)+\omega_{2}\left(\frac{1}{2}\right) \int_{0}^{1} G_{2}(\tau, x) q(x) d x\right] d \tau \\
& =c_{2} D c \eta_{0},
\end{aligned}
$$

Hence, for $u \in D_{1} \cap K, \alpha(u)=c$, we get

$$
\alpha(T u)=\|T u\|_{0}+\left\|(T u)^{\prime \prime}\right\|_{0}<B c \eta_{0}+c_{2} D c \eta_{0}=\left(B+c_{2} D\right) c \eta_{0}=c .
$$

Whereas for $u \in D_{2} \cap K, \alpha(u)=b$, there is $\|u\|_{0} \geq \frac{b}{2}$ or $\left\|u^{\prime \prime}\right\|_{0} \geq \frac{b}{2}$, By Lemma 2.4, we get

$$
\min _{\frac{1}{4} \leq t \leq \frac{3}{4}} u(t) \geq d_{1}\|u\|_{0} \geq \frac{d_{1} b}{2} \text { or } \min _{\frac{1}{4} \leq t \leq \frac{3}{4}}\left(-u^{\prime \prime}(t)\right) \geq \frac{d_{2} \xi}{c_{2}}\left\|u^{\prime \prime}\right\|_{0} \geq \frac{d_{2} \xi b}{2 c_{2}} .
$$

Therefore, using $\left(H_{4}\right)$ and (3.3), we have

$$
\begin{aligned}
\left|(T u)^{\prime \prime}\left(\frac{1}{2}\right)\right| & =\left|\lambda \int_{0}^{1} Q_{2}\left(\frac{1}{2}, \tau\right) f_{1}\left(\tau, u(\tau), u^{\prime}(\tau), u^{\prime \prime}(\tau), u^{\prime \prime \prime}(\tau)\right) d \tau\right| \\
& >\left|\lambda \int_{\frac{1}{4}}^{\frac{3}{4}} Q_{2}\left(\frac{1}{2}, \tau\right) f_{1}\left(\tau, u(\tau), u^{\prime}(\tau), u^{\prime \prime}(\tau), u^{\prime \prime \prime}(\tau)\right) d \tau\right| \\
& \geq \lambda \times \frac{b \eta_{1}}{\lambda} \int_{\frac{1}{4}}^{\frac{3}{4}} Q_{2}\left(\frac{1}{2}, \tau\right) d \tau \\
& =b .
\end{aligned}
$$

Hence,

$$
\alpha(T u) \geq\left|(T u)^{\prime \prime}\left(\frac{1}{2}\right)\right|>b .
$$


By (3.2), (3.4), and $\left(H_{5}\right)$, for $u \in K$, we have

$$
\begin{aligned}
\left\|(T u)^{\prime}\right\|_{0}= & \max _{t \in[0,1]} \mid \lambda \int_{t}^{1} \int_{0}^{1} Q_{2}(s, \tau) f_{1}\left(\tau, u(\tau), u^{\prime}(\tau), u^{\prime \prime}(\tau), u^{\prime \prime \prime}(\tau)\right) d \tau d s \\
& -\lambda \int_{0}^{1} \int_{0}^{1} s Q_{2}(s, \tau) f_{1}\left(\tau, u(\tau), u^{\prime}(\tau), u^{\prime \prime}(\tau), u^{\prime \prime \prime}(\tau)\right) d \tau d s \mid \\
\leq & \max _{t \in[0,1]}\left|\lambda \int_{t}^{1} \int_{0}^{1} Q_{2}(s, \tau) f_{1}\left(\tau, u(\tau), u^{\prime}(\tau), u^{\prime \prime}(\tau), u^{\prime \prime \prime}(\tau)\right) d \tau d s\right| \\
& +\max _{t \in[0,1]}\left|\lambda \int_{0}^{1} \int_{0}^{1} s Q_{2}(s, \tau) f_{1}\left(\tau, u(\tau), u^{\prime}(\tau), u^{\prime \prime}(\tau), u^{\prime \prime \prime}(\tau)\right) d \tau d s\right| \\
\leq & \lambda\left|\int_{0}^{1} \int_{0}^{1}(1+s) Q_{2}(s, \tau) f_{1}\left(\tau, u(\tau), u^{\prime}(\tau), u^{\prime \prime}(\tau), u^{\prime \prime \prime}(\tau)\right) d \tau d s\right| \\
\leq & \lambda \times \frac{\eta_{2} L}{\lambda}\left|\int_{0}^{1} \int_{0}^{1}(1+s)\left[c_{2} G_{2}(\tau, \tau)+\omega_{2}\left(\frac{1}{2}\right) \int_{0}^{1} G_{2}(\tau, x) q(x) d x\right] d \tau d s\right| \\
\leq & \eta_{2} L \times \frac{3}{2} c_{2} \int_{0}^{1}\left[G_{2}(\tau, \tau)+\omega_{2}\left(\frac{1}{2}\right) \int_{0}^{1} G_{2}(\tau, x) q(x) d x\right] d \tau \\
= & \frac{3}{2} c_{2} D \eta_{2} L, \\
\left\|(T u)^{\prime \prime \prime}\right\|_{0}= & \max _{t \in[0,1]}\left|\lambda \int_{0}^{1} \frac{\partial Q_{2}(t, \tau)}{\partial t} f_{1}\left(\tau, u(\tau), u^{\prime}(\tau), u^{\prime \prime}(\tau), u^{\prime \prime \prime}(\tau)\right) d \tau\right| \\
\leq & 2 \lambda \int_{0}^{1}\left[\frac{\sin \sqrt{A} \tau}{\sin \sqrt{A}}+\frac{\sqrt{A} \int_{0}^{1} G_{2}(\tau, x) q(x) d x}{\sin \sqrt{A}-\int_{0}^{1} q(x) \sin \sqrt{A} x d x-\int_{0}^{1} q(x) \sin \sqrt{A}(1-x) d x}\right] \\
& \times\left|f_{1}\left(\tau, u(\tau), u^{\prime}(\tau), u^{\prime \prime}(\tau), u^{\prime \prime \prime}(\tau)\right)\right| d \tau \\
& <\lambda 2 F \times \frac{\eta_{2} L}{\lambda} \\
& =2 F \eta_{2} L .
\end{aligned}
$$

So,

$$
\beta(T u)=\left\|(T u)^{\prime}\right\|_{0}+\left\|(T u)^{\prime \prime \prime}\right\|_{0}<\frac{3}{2} c_{2} D \eta_{2} L+2 F \eta_{2} L=\left(\frac{3}{2} c_{2} D+2 F\right) \eta_{2} L=L .
$$

Theorem 2.1 implies there is $\left(\Omega_{2} \backslash \bar{\Omega}_{1}\right) \cap K$ such that $u=T u$. So, $u(t)$ is a positive solution for BVP (1.1) satisfying

$$
c<\alpha(u)<b, \quad \beta(u)<L .
$$

Thus, Theorem 3.1 is completed.

\section{Example}

Example 4.1. Consider the following boundary value problem

$$
\left\{\begin{array}{l}
u^{(4)}(t)+\frac{\pi^{2}}{9} u^{\prime \prime}(t)=\pi^{2} f\left(t, u(t), u^{\prime}(t), u^{\prime \prime}(t), u^{\prime \prime \prime}(t)\right), \quad 0<t<1, \\
u(0)=u(1)=\int_{0}^{1} s u(s) d s \\
u^{\prime \prime}(0)=u^{\prime \prime}(1)=0
\end{array}\right.
$$


where

$$
f\left(t, u, v, u_{0}, v_{0}\right)=\left\{\begin{array}{l}
\frac{1}{20}\left(u-u_{0}\right)+\frac{1}{2}\left|\cos \left(v+v_{0}\right)\right|, \\
\quad\left(t, u, v, u_{0}, v_{0}\right) \in[0,1] \times[0,2] \times[-16000,16000] \times[-2,0] \times[-16000,16000], \\
\frac{1}{20}\left(2-u_{0}\right)(3-u)+\frac{27}{2}\left(3-u_{0}\right)(u-2)+\frac{1}{2}\left|\cos \left(v+v_{0}\right)\right|, \\
\quad\left(t, u, v, u_{0}, v_{0}\right) \in[0,1] \times[2,3] \times[-16000,16000] \times[-2,0] \times[-16000,16000], \\
\frac{1}{20}(u+2)\left(u_{0}+3\right)-\frac{27}{2}(u+3)\left(u_{0}+2\right)+\frac{1}{2}\left|\cos \left(v+v_{0}\right)\right|, \\
\quad\left(t, u, v, u_{0}, v_{0}\right) \in[0,1] \times[0,2] \times[-16000,16000] \times[-3,-2] \times[-16000,16000], \\
\frac{1}{5}(3-u)\left(u_{0}+3\right)+\frac{135}{2}(u-2)\left(u_{0}+3\right)-\frac{27}{2}(u+3)\left(u_{0}+2\right)+\frac{1}{2}\left|\cos \left(v+v_{0}\right)\right|, \\
\left(t, u, v, u_{0}, v_{0}\right) \in[0,1] \times[2,3] \times[-16000,16000] \times[-3,-2] \times[-16000,16000], \\
\frac{27}{2}\left(u-u_{0}\right)+\frac{1}{2}\left|\cos \left(v+v_{0}\right)\right|, \\
\quad\left(t, u, v, u_{0}, v_{0}\right) \in[0,1] \times[3,40] \times[-16000,16000] \times[-40,0] \times[-16000,16000], \\
\quad U[0,1] \times[0,40] \times[-16000,16000] \times[-40,-3] \times[-16000,16000] .
\end{array}\right.
$$

In this problem, we know that $A=\frac{\pi^{2}}{9}, \lambda=\pi^{2}, p(t)=t, q(t)=0$, then we can get $b_{1}=1, b_{2}=\frac{\sqrt{3} \pi}{6}, c_{1}=1, c_{2}=\frac{2 \sqrt{3}}{3}, \omega_{1}=2, \omega_{2}=\frac{2 \sqrt{3} \sin \frac{\pi}{3}(1+t)}{3}, d_{1}=\frac{3}{16}, d_{2}=\frac{\sqrt{3}-1}{4}, \xi=\frac{\sqrt{2+\sqrt{3}}}{2}$. Further more, we obtain

$$
B=\frac{1944 \sqrt{3}-972 \pi-9 \pi^{3}}{4 \pi^{5}}, \quad D=\frac{9-\sqrt{3} \pi}{2 \pi^{2}}, \quad F=\frac{\sqrt{3}}{\pi} .
$$

then $\quad \eta_{0}=\frac{12 \pi^{5}}{5832 \sqrt{3}-2916 \pi-27 \pi^{3}+36 \sqrt{3} \pi^{3}-12 \pi^{4}}, \quad \eta_{1}=\frac{\pi^{2}}{3 \sqrt{6+3 \sqrt{3}}-9}$,

$\theta=\min \left\{\frac{d_{1}}{2}, \frac{d_{2} \xi}{2 c_{2}}\right\}=\frac{\sqrt{2+\sqrt{3}}(3-\sqrt{3})}{32}, \quad \theta=\min \left\{\frac{d_{1}}{2}, \frac{d_{2} \xi}{2 c_{2}}\right\}=\frac{\sqrt{2+\sqrt{3}}(3-\sqrt{3})}{32}$,

$\theta b \approx 3.06>3$.

If we take $c=2, b=40, L=16000$, then we get

$f\left(t, u, v, u_{0}, v_{0}\right)=\frac{1}{20}\left(u-u_{0}\right)+\frac{1}{2}\left|\cos \left(v+v_{0}\right)\right| \leq 0.7<\frac{c \eta_{0}}{\lambda} \approx 0.8$

for $\left(t, u, v, u_{0}, v_{0}\right) \in[0,1] \times[0,2] \times[-16000,16000] \times[-2,0] \times[-16000,16000]$,

$f\left(t, u, v, u_{0}, v_{0}\right)=\frac{27}{2}\left(u-u_{0}\right)+\frac{1}{2}\left|\cos \left(v+v_{0}\right)\right| \geq 40>\frac{b \eta_{1}}{\lambda} \approx 38$,

for $\left(t, u, v, u_{0}, v_{0}\right) \in\left[\frac{1}{4}, \frac{3}{4}\right] \times[\theta b, 40] \times[-16000,16000] \times[-40,0] \times[-16000,16000]$

$$
\cup\left[\frac{1}{4}, \frac{3}{4}\right] \times[0,40] \times[-16000,16000] \times[-40,-\theta b] \times[-16000,16000],
$$

$f\left(t, u, v, u_{0}, v_{0}\right) \leq 1080.5<\frac{L \eta_{2}}{\lambda} \approx 1146$,

for $\left(t, u, v, u_{0}, v_{0}\right) \in[0,1] \times[0,40] \times[-16000,16000] \times[-40,0] \times[-16000,16000]$.

Then all the conditions of Theorem 3.1 are satisfied. Therefore, by Theorem 3.1 we know that boundary value problem (4.1) has at least one positive solution $u(t)$ satisfying

$$
2<\alpha(u)<40, \beta(u)<16000 .
$$

\section{Acknowledgements}

The project is supported by the Natural Science Foundation of China (10971045) and the Natural Science Foundation of Hebei Province (A2009000664, A2011208012). The research item financed by the talent training project funds of Hebei Province. The authors would like to thank the referee for helpful comments and suggestions. 


\section{Author details}

${ }^{1}$ College of Electrical Engineering and Information, Hebei University of Science and Technology, Shijiazhuang 050018, Hebei, P. R. China ${ }^{2}$ College of Sciences, Hebei University of Science and Technology, Shijiazhuang 050018, Hebei, P. R. China

\section{Authors' contributions}

The authors declare that the work was realized in collaboration with same responsibility.

All authors read and approved the final manuscript.

\section{Competing interests}

The authors declare that they have no competing interests.

Received: 29 September 2011 Accepted: 2 March 2012 Published: 2 March 2012

\section{References}

1. Bai, ZB: The method of lower and upper solution for a bending of an elastic beam equation. J Math Anal Appl. 248, 195-202 (2000). doi:10.1006/jmaa.2000.6887

2. Bai, ZB: The method of lower and upper solutions for some fourth-order boundary value problems. Nonlinear Anal. 67, 1704-1709 (2007). doi:10.1016/.na.2006.08.009

3. Chai, GQ: Existence of positive solutions for fourth-order boundary value problem with variable parameters. Nonlinear Anal. 66, 870-880 (2007). doi:10.1016/j.na.2005.12.028

4. Zhao, JF, Ge, WG: Positive solutions for a higher-order four-point boundary value problem with a $p$-Laplacian. Comput Math Appl. 58, 1103-1112 (2009). doi:10.1016/..camwa.2009.04.022

5. Bai, ZB: Positive solutions of some nonlocal fourth-order boundary value problem. Appl Math Comput. 215, 4191-4197 (2010). doi:10.1016/j.amc.2009.12.040

6. Li, YX: Positive solutions of fourth-order boundary value problems with two parameters. J Math Anal Appl. 281, 477-484 (2003). doi:10.1016/50022-247X(03)00131-8

7. $\mathrm{Ma}$, HL: Symmetric positive solutions for nonlocal boundary value problems of fourth-order. Nonlinear Anal. 68 , 645-651 (2008). doi:10.1016/j.na.2006.11.026

8. Ma, RY: Existence of positive solutions of a fourth-order boundary value problem. Appl Math Comput. 168, 1219-1231 (2005). doi:10.1016/j.amc.2004.10.014

9. $\mathrm{Ma}, \mathrm{R}$, Wang, $\mathrm{H}$ : On the existence of positive solutions of fourth-order ordinary differential equations. Appl Anal. 59 225-231 (1995). doi:10.1080/00036819508840401

10. Yao, QL: Local existence of multiple positive solutions to a singular cantilever beam equation. J Math Anal Appl. 363 , 138-154 (2010). doi:10.1016/j.jmaa.2009.07.043

11. Guo, D, Lakshmikantham, V: Nonlinear Problems in Abstract Cones. Academic Press, New York (1988)

12. Guo, YP, Ge, WG: Positive solutions for three-point boundary value problems with dependence on the first order derivatives. J Math Anal Appl. 290, 291-301 (2004). doi:10.1016/j.jmaa.2003.09.061

doi:10.1186/1687-2770-2012-29

Cite this article as: Guo et al.: Positive solutions for nonlocal fourth-order boundary value problems with all order derivatives. Boundary Value Problems 2012 2012:29.

\section{Submit your manuscript to a SpringerOpen ${ }^{\circ}$ journal and benefit from:}

- Convenient online submission

- Rigorous peer review

- Immediate publication on acceptance

- Open access: articles freely available online

- High visibility within the field

- Retaining the copyright to your article

Submit your next manuscript at $\boldsymbol{\wedge}$ springeropen.com 